\title{
Ensemble-Based Variational Method for Nonlinear Inversion of Surface Gravity Waves
}

\author{
WATARU FUJIMOTOa ${ }^{\mathrm{a}}$ AND TAKUJI WASEDA \\ Graduate School of Frontier Sciences, The University of Tokyo, Kashiwa, Chiba, Japan
}

(Manuscript received 26 April 2019, in final form 11 September 2019)

\begin{abstract}
Freak/rogue waves are considered to be the causes of marine accidents and their generation mechanism is closely related to the formation of wave groups. However, observations that capture the spatiotemporal evolution of coherent wave groups in directional windsea are rather limited. The paper presents a new technique known as the surface wave reconstruction by ensemble adjoint-free data assimilation (SWEAD) method that enables reconstruction of a spatiotemporal wave field covering a large area from wave records limited in observational density and spatial extent. We reconstructed spatiotemporal profiles of nonlinear surface gravity waves from virtual observational data using the adjoint-free four-dimensional variational data assimilation (a4DVar) scheme. The higher-order spectral method (HOSM) is used as a forward deepwater nonlinear wave model in a realistic sea state. The a4DVar scheme uses perturbed ensemble simulations to calculate the cost function gradient and Hessian; thus, construction of an adjoint model is not needed. A few extensions of the a4DVar scheme are proposed in this study. For efficient wave reconstruction, perturbed ensemble simulation results are reused by increasing the searching direction dimension at each iteration while assuring conformity to the perturbed model's linearity. For regularization, Fourier coefficient magnitudes are constrained by a known power spectrum from the phase-averaged wave model. Twin experiments were conducted for a unidirectional wave with virtual wave gauge data and a multidirectional wave with virtual stereo camera imaging data. For both unidirectional and multidirectional cases, nonlinear freak wave-related wave groups were well reproduced, which is impossible using a linear model.
\end{abstract}

\section{Introduction}

Freak waves with wave heights significantly exceeding the significant wave height (e.g., $H>2 H_{m 0}$ ) pose a risk for ships and offshore structures (Kharif and Pelinovsky 2003). Despite these risks, space and time $(2 D+T)$ observations of freak waves has been limited. Freak waves have been observed in the ocean (e.g., Haver 2004; Waseda et al. 2011; Magnusson and Donelan 2013; Flanagan et al. 2016); however, most measurements have been taken by moored buoys or wave gauges on a tower, thus providing only a time series. Stereo camera imaging is a promising alternative for $2 D+T$ freak wave observations (Benetazzo et al. 2017), but it has a measurable area limited to a

\footnotetext{
${ }^{a}$ Current affiliation: MS\&AD InterRisk Research and Consulting, Inc., Tokyo, Japan.
}

Corresponding author: Takuji Waseda, waseda@k.u-tokyo.ac.jp few wavelengths. Because of this, it is still currently difficult to directly observe a freak wave's spatiotemporal evolution. This study presents a technique for reconstructing freak wave spatiotemporal profiles from limited observational data.

Reconstructing a $2 D+T$ wave field in a large domain may be crucial to pinning down the mechanism that generates freak waves. Two freak wave generation mechanisms have been proposed: nonlinear focusing by four-wave quasi resonance (Zakharov 1968; Janssen 2003) and linear-phase focusing (Kharif and Pelinovsky 2003). Fujimoto et al. (2019) investigated possible mechanisms of two freak waves observed in the Pacific Ocean and demonstrated that the freak waves deformed due to nonlinear four-wave quasi resonance. Such deformation causes freak wave asymmetry, which intensifies wave loads on ships and may cause maritime accidents (Fonseca et al. 2006; Guedes Soares et al. 2008; Houtani et al. 2016). Therefore, nonlinear inversion to reproduce freak wave evolution 
beyond the observational domain is imperative for reducing maritime risks.

Variable current fields (White and Fornberg 1998; Toffoli et al. 2013, 2015) and variable winds (Annenkov and Shrira 2009; Toffoli et al. 2017) are also known to cause and affect the freak wave generation. One of these effects might be a crucial factor in the generation of a particular event, but in the end, the lifetime of the freak wave group is related to the nonlinearity (Fujimoto et al. 2019). The focus of this study is to merge the nonlinear wave model and the observational data to study the long-term evolution of the freak wave group by means of data assimilation.

Studies have proposed a sequential method to assimilate radar data to a wave model at a fast computational speed for real-time deterministic wave forecasts (Yoon et al. 2015; Wijaya et al. 2015; van Groesen and Wijaya 2017). In contrast, to exploit spatially limited observational data, we adopted inversion based on a variational principle to assure physical consistency of nonlinear freak waves in a relatively long assimilation window.

The nonlinear Schrödinger equation (NLS) has been employed for wave reconstruction based on observed time records (Slunyaev et al. 2013). The NLS captures modulational instability's essential physics; however, it is valid only under a narrowband assumption (Zakharov 1968). In contrast, the higher-order spectral method (HOSM; Dommermuth and Yue 1987; West et al. 1987) has no spectral bandwidth limitation, making it a suitable forward model for variational data assimilation. The HOSM is a type of direct numerical simulation (DNS) for surface waves that efficiently solves the Euler equation to an arbitrary order of nonlinearity. The HOSM is becoming a standard tool for computing phase-resolved wave fields from a given directional spectrum (Onorato et al. 2002; Socquet-Juglard et al. 2005; Gramstad and Trulsen 2007; Toffoli and BitnerGregersen 2011; Xiao et al. 2013).

$\mathrm{Wu}(2004)$ and Blondel-Couprie et al. (2010, 2013) applied a four-dimensional variational data assimilation (4DVar) method to the HOSM using wave gauge data to estimate a spatial wave field. This method required many iterations because the Rosenbrock method, a derivative-free optimization technique, was adopted to minimize squared error between observations and model predictions. Aragh and Nwogu (2008) successfully applied an adjoint method for the one-dimensional HOSM to the third order of nonlinearity, and further extended it to a two-dimensional problem (Aragh et al. 2008). However, extending this adjoint method to a higher-order two-dimensional HOSM is cumbersome as the number of terms involved increases at an accelerating rate. An additional difficulty in implementing the adjoint code is associated with the breaking dissipation parameterization of HOSM (Tian et al. 2010). With the truncated nonlinearity and the single-valued free surface, HOSM computation fails when waves undergo energetic plunging breaking. To continue the computation beyond such an event, the localized and conditional filter is applied. Such physical parameterization of the subgrid process is known to cause difficulty in constructing an adjoint code (Zhu et al. 2002). Last, the task of constructing a parallel code, that requires high-level programming skill and maintenance burden, is a practical issue leading to human error.

To circumvent convergence speed issues and coding difficulties, we employed the ensemble-based 4DVar in this study. The cost function's gradient is computed using perturbed ensemble simulations, making adjoint code unnecessary. In contrast to the adjoint method, increasing the HOSM simulation's nonlinearity is straightforward. Section 2 explains 4DVar ensemble method advantages over the adjoint method. We applied adjoint-free 4DVar (a4DVar) (Yaremchuk et al. 2017a, hereafter Y17) with some modifications for wave reconstruction. Section 3 describes wave inversion implementation. We tested the proposed method using twin-data experiments for unidirectional and multidirectional cases in section 4. Last, conclusions and possible future study directions are offered in section 5 .

\section{Adjoint-free 4DVar method}

To distinguish between the state vectors and data space vectors, in what follows we will denote them by boldface Arial and boldface Times New Roman fonts, respectively. The $4 \mathrm{DV}$ ar cost function is formulated as ordinary least squares:

$$
\begin{aligned}
J\left(\mathbf{x}_{0}\right)= & \frac{1}{2}\left[\mathbf{H}\left(\mathbf{x}_{0}\right)-\mathbf{y}\right]^{\mathrm{T}} \mathbf{R}^{-1}\left[\mathbf{H}\left(\mathbf{x}_{0}\right)-\mathbf{y}\right] \\
& +\frac{1}{2}\left(\mathbf{x}_{0}-\mathbf{x}_{b}\right)^{\mathrm{T}} \mathbf{B}^{-1}\left(\mathbf{x}_{0}-\mathbf{x}_{b}\right),
\end{aligned}
$$

where $\mathbf{H}\left(\mathbf{x}_{0}\right)$ is the model-data nonlinear projection operator, $\mathbf{y}$ is the observational data vector, and $\mathbf{R}$ is the observation error covariance. The background error covariance matrix $\mathbf{B}$ represents error statistics of initial condition $\mathbf{x}_{0}$ in the Gaussian statistic framework.

\section{a. Overview of the ensemble-based 4DVar}

The cost function gradient in an ensemble-based 4DVar can be expressed as 


$$
\boldsymbol{\nabla} J=\mathbf{H}^{\mathrm{T}} \mathbf{R}^{-1}\left[\mathbf{H}\left(\mathbf{x}_{0}\right)-\mathbf{y}\right]+\mathbf{B}^{-1}\left(\mathbf{x}_{0}-\mathbf{x}_{b}\right),
$$

where $\nabla$ denotes differentiation by the control variable, initial condition $\mathbf{x}_{0}$. The adjoint matrix $\mathbf{H}^{\mathrm{T}}$ of the Jacobian matrix $\mathbf{H}$ of the model-data projection operator $\mathbf{H}\left(\mathbf{x}_{0}\right)$ is necessary to compute the cost function gradient in Eq. (2). Iterative gradient methods such as the conjugate gradient or quasi-Newton method have conventionally been used to update $\mathbf{x}_{0}$ iteratively, thus minimizing the cost function. However, it is laborious to program an adjoint code to construct $\mathbf{H}^{\mathrm{T}}$. Moreover, the tangent linear approximation fails because of the physical system's strong nonlinearity (see section 1).

Instead of needing an adjoint code, the ensemblebased 4DVar uses the cost function gradient and Jacobian matrix $\mathbf{H}$, which are computed using perturbed ensemble simulations. We let $\mathbf{p}_{m}\left(m=1, \ldots, N_{\text {ens }}\right)$ be the perturbation vector of initial condition $\mathbf{x}_{0}$, where $N_{\text {ens }}$ is the number of ensembles. The model increment $\boldsymbol{\delta} \mathbf{Y}_{m}$ for each perturbation is

$$
\boldsymbol{\delta} \mathbf{Y}_{m} \equiv \mathbf{H}\left(\mathbf{x}_{0}+\mathbf{p}_{m}\right)-\mathbf{H}\left(\mathbf{x}_{0}\right)=\mathbf{H} \mathbf{p}_{m}+O\left(\left\|\mathbf{p}_{m}\right\|^{2}\right) .
$$

If perturbation vectors are sufficiently small, the higherorder term $O\left(\left\|\mathbf{p}_{m}\right\|^{2}\right)$ is negligible. When perturbation vectors $\mathbf{p}_{m}$ and $\boldsymbol{\delta} \mathbf{Y}_{m}$ are assembled in matrices $\mathbf{P}=$ $\left(\begin{array}{lll}\cdots & \mathbf{p}_{m} & \cdots\end{array}\right)$ and $\boldsymbol{\delta} \mathbf{Y}=\left(\begin{array}{llll}\cdots & \boldsymbol{\delta} \mathbf{Y}_{m} & \cdots\end{array}\right)$, Eq. (3) can thus be reduced to

$$
\delta \mathbf{Y} \approx \mathrm{HP} .
$$

From Eqs. (2) and (4) we obtain the expression for the cost function gradient in a subspace spanned by $\mathbf{P}$ :

$$
\mathbf{P}^{\mathrm{T}} \boldsymbol{\nabla} J \approx \boldsymbol{\delta} \mathbf{Y}^{\mathrm{T}} \mathbf{R}^{-1}\left[\mathbf{H}\left(\mathbf{x}_{0}\right)-\mathbf{y}\right]+\mathbf{P}^{\mathrm{T}} \mathbf{B}^{-1}\left(\mathbf{x}_{0}-\mathbf{x}_{b}\right) .
$$

\section{b. Review of the adjoint-free 4DVar}

There are ensemble-based method variants that use different ensemble generation approaches and optimization schemes. The adjoint-free 4DVar uses empirical orthogonal function (EOF) modes of the model trajectory and/or misfits between the model and observations to generate ensemble members (Panteleev et al. 2015; Yaremchuk et al. 2016; Y17; Yaremchuk et al 2017b). En4DVar (Liu et al. 2008, 2009) utilizes meteorological forecasting ensemble members. The maximum likelihood ensemble filter (MLEF; Zupanski 2005) uses ensemble Kalman filter members to optimize the cost function, whereas a4DVar does not rely on an ensemble forecast and rather estimates the background error covariance through heuristic means (e.g., a heat transfer model). In addition, a4DVar is applicable even if data are limited, which would cause the ordinary 4DVar to experience instability (Yaremchuk et al. 2009, 2016; Y17). Therefore, we have adopted a4DVar for wave reconstruction.

To update control variable $\mathbf{x}_{0}$, a4DVar relies on linear superposition of the perturbation vectors, $\mathbf{x}_{0}+$ Ps. Here $\mathbf{s} \in \mathbb{R}^{N_{\text {ens }}}$ is the update's weighting coefficients and is yet to be determined. Equation (5) is rewritten replacing $\mathbf{x}_{0}$ with $\mathbf{x}_{0}+$ Ps:

$$
\begin{aligned}
\mathbf{P}^{\mathrm{T}} \boldsymbol{\nabla} J & =\mathbf{P}^{\mathrm{T}} \mathbf{H}^{\mathrm{T}} \mathbf{R}^{-1}\left[\mathbf{H}\left(\mathbf{x}_{0}+\mathbf{P s}\right)-\mathbf{y}\right]+\mathbf{P}^{\mathrm{T}} \mathbf{B}^{-1}\left(\mathbf{x}_{0}+\mathbf{P s}-\mathbf{x}_{b}\right) \\
& =\mathbf{P}^{\mathrm{T}} \mathbf{H}^{\mathrm{T}} \mathbf{R}^{-1}\left[\mathbf{H}\left(\mathbf{x}_{0}\right)+\mathbf{H P s}-\mathbf{y}\right]+\mathbf{P}^{\mathrm{T}} \mathbf{B}^{-1}\left(\mathbf{x}_{0}+\mathbf{P s}-\mathbf{x}_{b}\right)+O\left(\|\mathbf{s}\|^{2}\right) \\
& \approx \boldsymbol{\delta} \mathbf{Y}^{\mathrm{T}} \mathbf{R}^{-1}\left[\mathbf{H}\left(\mathbf{x}_{0}\right)+\boldsymbol{\delta} \mathbf{Y} \mathbf{s}-\mathbf{y}\right]+\mathbf{P}^{\mathrm{T}} \mathbf{B}^{-1}\left(\mathbf{x}_{0}+\mathbf{P s}-\mathbf{x}_{b}\right)+O\left(\|\mathbf{s}\|^{2}\right) .
\end{aligned}
$$

Weighting coefficient $\mathbf{s}$ can be determined from the condition that the cost function gradient is null at the next iteration, $\nabla J=0$. We thus obtain

$$
\begin{aligned}
\left(\boldsymbol{\delta} \mathbf{Y}^{\mathrm{T}} \mathbf{R}^{-1} \boldsymbol{\delta} \mathbf{Y}+\mathbf{P}^{\mathrm{T}} \mathbf{B}^{-1} \mathbf{P}\right) \mathbf{s}= & \boldsymbol{\delta} \mathbf{Y}^{\mathrm{T}} \mathbf{R}^{-1}\left[\mathbf{y}-\mathbf{H}\left(\mathbf{x}_{0}\right)\right] \\
& -\mathbf{P}^{\mathrm{T}} \mathbf{B}^{-1}\left(\mathbf{x}_{0}-\mathbf{x}_{b}\right) .
\end{aligned}
$$

Solving Eq. (7) is straightforward because $\delta \mathbf{Y}^{\mathrm{T}} \mathbf{R}^{-1} \boldsymbol{\delta} \mathbf{Y}$ is a square matrix of dimension $N_{\mathrm{ens}} \times N_{\mathrm{ens}}$ and is not very large. Equation (7) corresponds to the GaussNewton method in that the Hessian matrix of cost function Eq. (1) is approximated by the product of the Jacobian matrix.

\section{c. Improving a4DVar: Reuse of the perturbation subset considering conformity to the linear approximation}

Efficient use of ensemble simulation results is crucial for high-dimensional problems. In the original a4DVar studies (Y17, and references therein), perturbed data in previous iterations were reused to increase search direction dimensions. The previous perturbation matrices $\mathbf{P}^{\text {pre }}$ and $\boldsymbol{\delta} \mathbf{Y}^{\text {pre }}$ were concatenated to new perturbation matrices $\mathbf{P}^{\text {new }}$ and $\boldsymbol{\delta} \mathbf{Y}^{\text {new }}$, [i.e., $\left(\mathbf{P}^{\text {pre }} \mid \mathbf{P}^{\text {new }}\right) \rightarrow \mathbf{P}$ and $\left.\left(\boldsymbol{\delta} \mathbf{Y}^{\text {pre }} \mid \boldsymbol{\delta} \mathbf{Y}^{\text {new }}\right) \rightarrow \boldsymbol{\delta} \mathbf{Y}\right]$. Concatenated perturbation data $\mathbf{P}$ and $\boldsymbol{\delta} \mathbf{Y}$ are used to find the optimal correction coefficient $\mathbf{s}$ in Eq. (7). 
The Jacobian matrix $\mathbf{H}$ of the model-data projection operator $\mathbf{H}\left(\mathbf{x}_{0}\right)$ varies with initial condition $\mathbf{x}_{0}$ because of nonlinearity of the physical and observational systems; $\boldsymbol{\delta} \mathbf{Y}^{\text {pre }}$ is a linear approximation of $\mathbf{H}\left(\mathbf{x}_{0}\right)$ around $\mathbf{x}_{0}$ of the previous iterations, and deviates from the approximation around $\mathbf{x}_{0}$ of the current iteration because $\mathbf{x}_{0}$ is updated during the optimization process. Previous studies (Yaremchuk et al. 2009; Panteleev et al. 2015; Yaremchuk et al. 2017b) proposed reinitializing perturbations $\mathbf{P}$ and $\boldsymbol{\delta} \mathbf{Y}$ at certain conditions defined for the cost function's decay rate (Yaremchuk et al. 2016) or the eigenvalues of $\boldsymbol{\delta} \mathbf{Y}^{\mathrm{T}} \mathbf{R}^{-1} \boldsymbol{\delta} \mathbf{Y}$ (Y17). Consequently, the concatenated perturbation matrix has a limited dimension because of this reinitialization. Moreover, those methods do not assure the accuracy of the linear approximation $\boldsymbol{\delta} \mathbf{Y} \approx \mathbf{H P}$.

We propose evaluating deviation from the linear approximation $\boldsymbol{\delta} \mathbf{Y} \approx \mathbf{H P}$ and retaining only perturbations that conform to the linear approximation. Deviation from the linear approximation is thus measured by comparing $\mathbf{s}^{n}$ and $\mathbf{s}^{n^{\prime}}$ :

$$
\begin{aligned}
\mathbf{s}^{n^{\prime}} \equiv & \left(\boldsymbol{\delta} \mathbf{Y}^{\mathrm{T}} \mathbf{R}^{-1} \boldsymbol{\delta} \mathbf{Y}+\mathbf{P}^{\mathrm{T}} \mathbf{B}^{-1} \mathbf{P}\right)^{-1} \\
& \times\left[\boldsymbol{\delta} \mathbf{Y}^{\mathrm{T}} \mathbf{R}^{-1}\left[\mathbf{H}\left(\mathbf{x}_{0}^{n+1}\right)-\mathbf{H}\left(\mathbf{x}_{0}^{n}\right)\right]+\mathbf{P}^{\mathrm{T}} \mathbf{B}^{-1} \mathbf{P}\right],
\end{aligned}
$$

where superscript $n$ represents the index of iterations. The details of Eq. (8)'s derivation are explained in the appendix. The subsets of concatenated perturbation matrices $\mathbf{P}$ and $\boldsymbol{\delta} \mathbf{Y}$ are extracted and are then reused as $\mathbf{P}^{\text {pre }}$ and $\boldsymbol{\delta} \mathbf{Y}^{\text {pre }}$ in the next iteration (Fig. 1). Note that Eq. (8) is applied to the entire concatenated matrices at every iteration. This procedure is called "Optimize" in Fig. 1. This formulation is derived from the secant condition linearly relating the Hessian matrix and cost function gradient [Eq. (1)]. If the physical and observational systems are linear, then $\mathbf{s}^{n}$ is equal to $\mathbf{s}^{n^{\prime}}$; however, nonlinearity in systems causes $\mathbf{s}^{n^{\prime}}$ to deviate from $\mathbf{s}^{n}$. The proposed method reuses perturbations if the normalized difference between $\mathbf{s}^{n}$ and $\mathbf{s}^{n^{\prime}}$ is less than the error tolerance $e_{\mathrm{TOL}}$ :

$$
\frac{\left|s_{i}^{n}-s_{i}^{n^{\prime}}\right|}{\sigma_{s^{n}}}<e_{\mathrm{TOL}},
$$

where $i=1, \ldots, N_{\mathrm{ens}}$ is the index assigned to elements in $\mathbf{s}^{n}$ and $\mathbf{s}^{n^{\prime}}$ and $\sigma_{s^{n}}$ denotes a standard deviation of $\mathbf{s}^{n^{\prime}}$. Perturbations $i=m_{1}, \ldots, m_{k}$ that satisfy the condition in Eq. (9) are extracted from concatenated perturbation matrices $\mathbf{P}$ and $\boldsymbol{\delta} \mathbf{Y}$, and error tolerance should thus be tuned for stable optimization.

In Eq. (9), $\mathbf{s}^{n}-\mathbf{s}^{n^{\prime}}$ is nonzero because of residual $O\left(\|\mathbf{s}\|^{2}\right)$ in Eq. (3) (see the appendix for more). Because $O\left(\|\mathbf{s}\|^{2}\right)$ decreases with iteration number $n, \mathbf{s}^{n^{\prime}}$ eventually

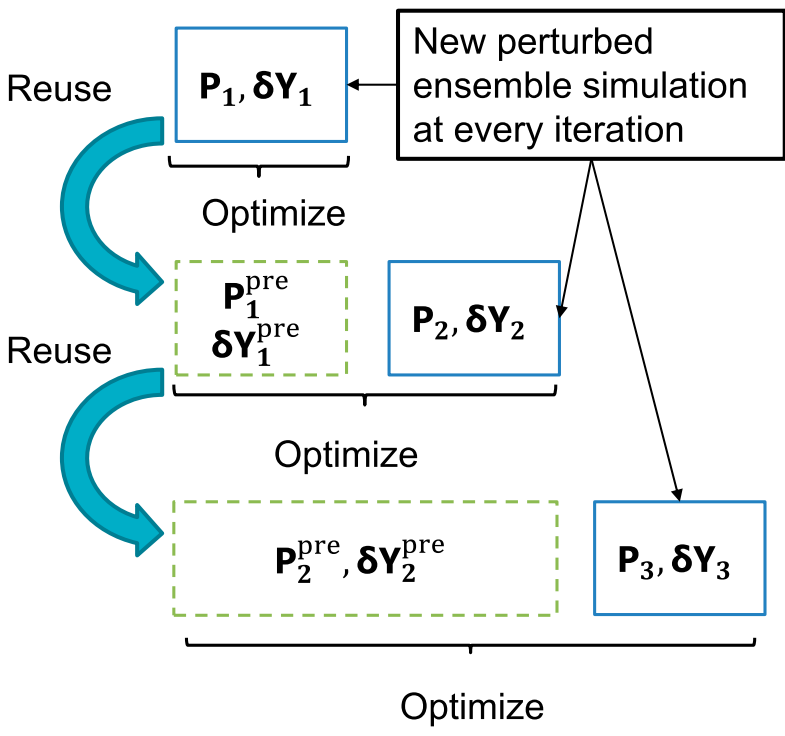

FIG. 1. Illustration of the procedure for reusing ensemble simulation results. Equation (8) is solved to obtain the optimal weighting coefficient $\mathbf{s}$ in the "Optimize" step. Then, only the perturbations satisfying the criteria in Eq. (9) are reused in the "Reuse" step. At each iteration, the number of additional perturbation vectors is fixed; however, the dimension of the perturbation matrix used for the optimization grows by reusing the previous iteration's perturbation matrix.

approaches $\mathbf{s}^{n}$; therefore, many perturbations satisfying Eq. (9) are reused later in the optimization.

Our proposed method has no fundamental limitation on the number of concatenated perturbations, that is, the optimization's subspace dimension. In principle, concatenated perturbation numbers can reach the model dimension; however, if the size of the concatenated ensembles $\delta \mathbf{Y}^{\mathrm{T}} \mathbf{R}^{-1} \boldsymbol{\delta} \mathbf{Y}$ is too large to keep in computer memory, more perturbation can be discarded.

\section{Application of the modified a4DVar for wave reconstruction}

We used the modified a4DVar for wave reconstruction with the HOSM. Section 3a explains the formulation of the cost function and control variable; section $3 \mathrm{~b}$ describes a regularization method using background power spectrum $S(\mathbf{k})$, which is estimated independently of the observation; and section $3 c$ discusses generation of ensemble members.

\section{a. Cost function for wave reconstruction}

The control variable $\mathbf{x}_{0}$ is the Fourier coefficient of surface elevation $\hat{\boldsymbol{\eta}}_{0}(\mathbf{k})$ in the wavenumber space. The background value $\mathbf{x}_{b}$ indicates averaged initial condition information and should be $\mathbf{x}_{b}=0$ under a random phase 
assumption. The wave reconstruction's cost function can be expressed as

$$
J\left(\hat{\boldsymbol{\eta}}_{0}\right)=\frac{1}{2}\left[\mathbf{H}\left(\hat{\boldsymbol{\eta}}_{0}\right)-\mathbf{y}\right]^{\mathrm{T}} \mathbf{R}^{-1}\left[\mathbf{H}\left(\hat{\boldsymbol{\eta}}_{0}\right)-\mathbf{y}\right]+\frac{1}{2} \hat{\boldsymbol{\eta}}_{0}^{*} \mathbf{B}^{-1} \hat{\boldsymbol{\eta}}_{0},
$$

where the asterisk $(*)$ denotes an adjoint vector.

Although surface elevation and velocity potential are necessary to describe water wave dynamics (Zakharov 1968), we adopted only surface elevation as a control variable for simplicity. The Fourier coefficients of the surface velocity potential are given as $\hat{\boldsymbol{\Phi}}_{0}(\mathbf{k})=$ $[i g / \omega(\mathbf{k})] \hat{\boldsymbol{\eta}}_{0}(\mathbf{k})$, assuming wave linearity, where $g$ is acceleration due to gravity and $\omega(\mathbf{k})$ is the angular frequency following the linear dispersion relation $\omega(\mathbf{k})=\sqrt{g|\mathbf{k}|}$. To assure physical consistency of analyzed waves, we applied a nonlinear spinup technique (Dommermuth 2000) to the HOSM simulation. Nonlinear terms in the HOSM gradually increase over time and the linear wave system gradually transforms to a nonlinear one with bound harmonics. The data assimilation window starts after nonlinear spinup (five wave periods in this study). This technique considers only free waves as the control variable, avoiding consideration of nonlinear bound wave generation.

\section{b. Background error covariance modeling for regularization}

The background error term is necessary to stabilize reconstruction, especially for limited amounts of observational data. This study utilizes a background power spectrum $S(\mathbf{k})$ obtained in advance of the wave reconstruction for modeling background error covariance. For example, output from a phase-averaged wave model such as WAVEWATCH III (Tolman et al. 2016) is a candidate for the background power spectrum. In a tank experiment, an input signal's power spectrum can be used as $S(\mathbf{k})$. The new technique constrains initialcondition amplitudes using the background power spectrum.

Because there should be no correlation between free wave components, the background error covariance matrix $\mathbf{B}$ is a diagonal matrix. The matrix's diagonal element is the variance of each wavenumber component, which should be proportional to the background power spectrum $S(\mathbf{k})$, that is, $\mathbf{B}_{i i}=S(\mathbf{k}) / \alpha$, where $i$ is an index of a wavenumber component, and $\alpha$ is the regularization parameter (see below). The cost function can thus be rewritten as

$$
J\left(\hat{\boldsymbol{\eta}}_{0}\right)=\frac{1}{2}\left[\mathbf{H}\left(\hat{\boldsymbol{\eta}}_{0}\right)-\mathbf{y}\right]^{\mathrm{T}} \mathbf{R}^{-1}\left[\mathbf{H}\left(\hat{\boldsymbol{\eta}}_{0}\right)-\mathbf{y}\right]+\frac{\alpha}{2} \hat{\boldsymbol{\eta}}_{0}^{*} \mathbf{B}^{\prime-1} \hat{\boldsymbol{\eta}}_{0},
$$

where $\mathbf{B}^{\prime}$ is a diagonal matrix $\mathbf{B}^{\prime}=\mathbf{B} / \alpha$. Therefore, $\mathbf{B}_{i i}^{\prime}=S\left(\mathbf{k}_{i}\right)$.

Modeling background error covariance is also known as Tikhonov regularization (Willoughby 1979). In this, $\alpha$ is the regularization parameter and should be tuned to balance between background and observational errors. Regularization parameter $\alpha$ should be large enough to give stable solutions; however, but smaller values for $\alpha$ yields yield less-biased solutions. Tuning of the regularization parameter $\alpha$ for twin experiments of this study is explained in sections $4 \mathrm{a}(2)$ and $4 \mathrm{~b}(2)$.

\section{c. Generating ensemble members from misfit power spectrum}

We chose appropriate perturbation modes to increase a4DVar's convergence speed. Previous studies utilized EOF modes of spatiotemporal model data to project misfit $\mathbf{y}_{t}-\mathbf{H}_{t}\left(\mathbf{x}_{t}\right)$ onto a physical model space (Panteleev et al. 2015; Yaremchuk et al. 2016, 2017b). For efficiency, only those EOF modes with large eigenvalues at each iteration were chosen as new ensemble members $\mathbf{P}^{\text {new }}$. The reason for using EOF modes is that ocean circulation may not necessarily be represented by the superposition of known harmonic functions. Therefore, empirical orthogonal modes were used. In contrast, surface waves are well represented by superposition of Fourier modes; therefore, we used Fourier modes instead of EOF modes as ensemble members in this study (Fig. 2). We determined the range of wavenumbers by selecting modes with large moduli of the misfit's $\mathbf{H}\left(\mathbf{x}_{0}\right)-\mathbf{y}$ Fourier modes. We then set the amplitude of perturbation Fourier modes $\mathbf{p}_{m}$ to $0.1 \%$ of the background power spectral amplitude $\sqrt{S(\mathbf{k})}$, satisfying condition $\left\|\mathbf{p}_{m}\right\|^{2} \ll 1$.

The original a4DVar (Yaremchuk et al. 2009; Panteleev et al. 2015) orthogonalizes $\mathbf{P}$ with respect to the Hessian matrix $\tilde{\mathbf{H}}=\mathbf{H}^{\mathrm{T}} \mathbf{R}^{-1} \mathbf{H}+\mathbf{B}^{-1}$ to avoid search directions that have been already explored and efficiently reuse perturbed data. In contrast, our proposed method does not require orthogonalization as the Fourier modes are approximately orthogonal with respect to the Hessian matrix $\tilde{\mathbf{H}}$, if correlations among $\boldsymbol{\delta} \mathbf{Y}_{m}$ can be ignored. We chose only those modes that are independent from the previous perturbation $\mathbf{P}^{\text {pre }}$ as new perturbations $\mathbf{P}^{\text {new }}$.

\section{Twin-data experiments}

Our proposed method combines a4DVar and the HOSM and reconstructs the evolving nonlinear wave field using the background power spectrum. We named this method surface wave reconstruction by ensemble adjointfree data assimilation (SWEAD). We conducted twin-data 


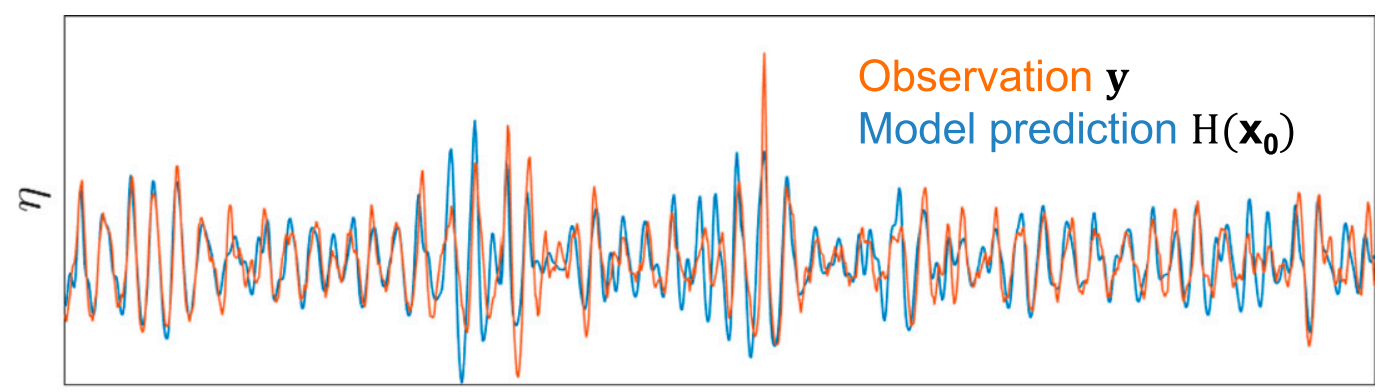

\section{Location or Time}

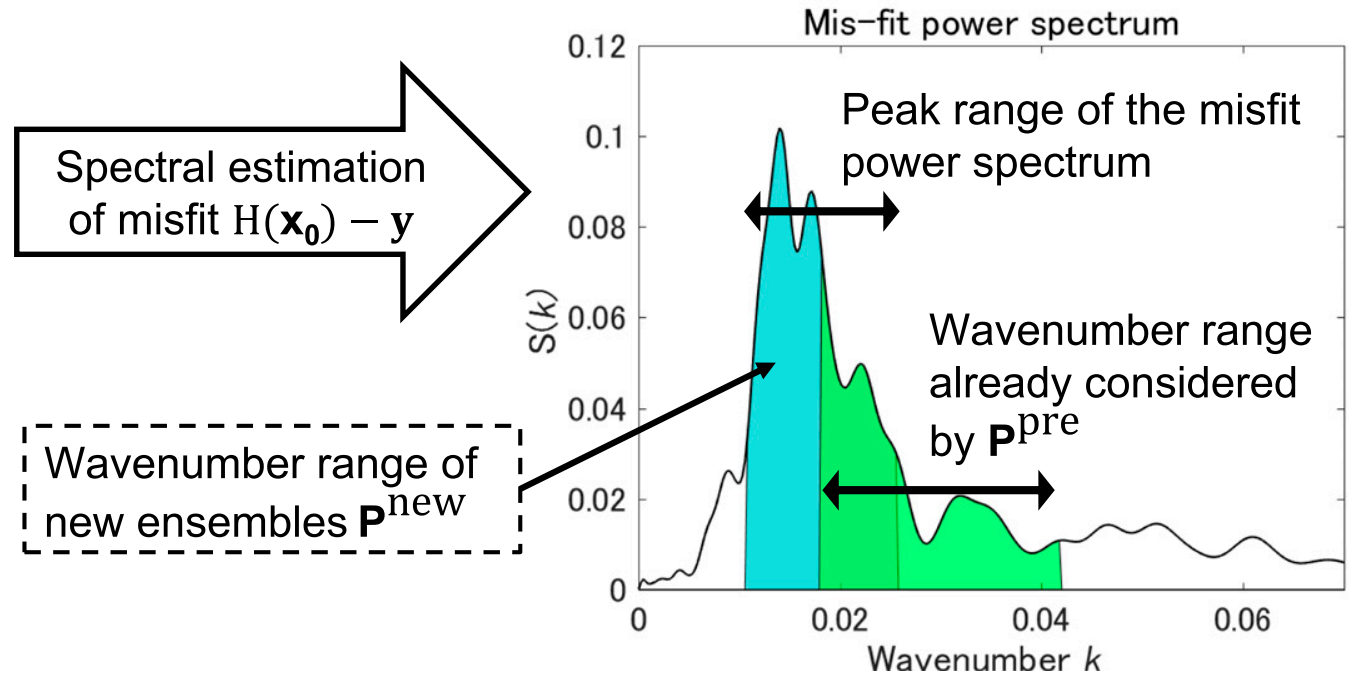

FIG. 2. Illustration of a procedure for generating new ensemble members considering the misfit. The range of wavenumbers around the misfit Fourier spectrum peak are first determined as new ensemble member candidates, and then those already considered by previous ensemble members $\mathbf{P}^{\text {pre }}$ are excluded.

experiments for unidirectional and multidirectional wave fields using the SWEAD method. Following the procedure outlined in Yaremchuk et al. (2009), we extracted virtual observational data from the true wave field of a HOSM simulation initialized using a given spectrum, and then used the same true wave field to validate the reconstructed wave field. The wave field is reconstructed using a HOSM model with a smaller domain that considers predictable distance based on ray theory. Section 4 a shows how the SWEAD method reconstructs the spatiotemporal wave profile in a unidirectional case. Section $4 \mathrm{~b}$ demonstrates the SWEAD method's applicability in a multidirectional case.

Virtual observational data is contaminated with white Gaussian noise with standard deviations of $10 \%, 30 \%$, and $50 \%$ of the true value's standard deviation. The cost function can then be simplified because the covariance matrix of observational error can be written as $\mathbf{R}=e^{2} \mathbf{I}$, where $e$ is white noise variance. Therefore, the cost function given in Eq. (11) can be rewritten as

$$
e^{2} J\left(\hat{\boldsymbol{\eta}}_{0}\right)=\frac{1}{2}\left\|\mathbf{H}\left(\hat{\boldsymbol{\eta}}_{0}\right)-\mathbf{y}\right\|^{2}+\frac{e^{2} \alpha}{2} \hat{\boldsymbol{\eta}}_{0}^{*} \mathbf{B}^{\prime-1} \hat{\boldsymbol{\eta}}_{0}
$$

which can be further simplified as follows:

$$
J\left(\hat{\boldsymbol{\eta}}_{0}\right)=\frac{1}{2}\left\|\mathbf{H}\left(\hat{\boldsymbol{\eta}}_{0}\right)-\mathbf{y}\right\|^{2}+\frac{\alpha}{2} \hat{\boldsymbol{\eta}}_{0}^{*} \mathbf{B}^{\prime-1} \hat{\boldsymbol{\eta}}_{0} .
$$

Here, $J\left(\hat{\boldsymbol{\eta}}_{0}\right)$ and $\alpha$ are normalized by $e^{2}$ and are thus redefined. The following sections show the results of the SWEAD method optimizing Eq. (13).

\section{a. Unidirectional wave twin experiment}

\section{1) Problem Settings}

We selected a true state containing a freak wave event from ensemble simulations of the HOSM conducted with a random initial wave field to spawn a freak wave considering third-order nonlinearity (Fig. 3). The initial wave field's power spectrum was the JONSWAP spectrum with peakedness factor $\gamma=3.3$ and steepness $H_{m 0} k_{p} / 2=0.11$. Here, $H_{m 0}$ is the significant wave height, 


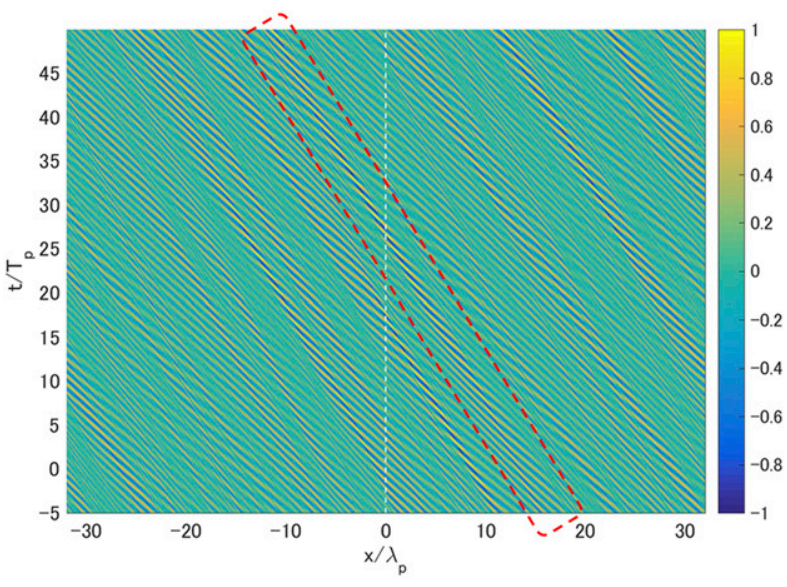

FIG. 3. Spatiotemporal profile of the true value $\eta_{t}$. This figure shows only the $64 \lambda_{p}$ region, but the generated true-value domain size was $128 \lambda_{p}$. The wave gauge was located at $\mathbf{x}=0$ (white broken line). The area surrounded by red broken lines indicates the freak wave group.

$k_{p}=2 \pi / \lambda_{p}$ is the peak wavenumber, and $\lambda_{p}$ is the peak wavelength. Sea conditions are relatively severe compared to those of the real sea (e.g., Bitner-Gregersen and Toffoli 2012). We set the spatial domain length to $128 \lambda_{p}$ to mitigate the effects of the periodic boundary condition assumed in the HOSM (Xiao et al. 2013). Time integration's temporal duration was $50 T_{p}$, with a nonlinear spinup time of $5 T_{p}$, where $T_{p}$ is the peak wave period. We set the time step to $\Delta t=T_{p} / 50$ and set the number of grids to capture wavenumber components up to $8 k_{p}$ (Tanaka and Yokoyama 2004).

The selected freak wave crest height is $\eta_{\max }=1.5 H_{m 0}$ (Fig. 4). We extracted a virtual observational wave gauge record from the true state at the location where the freak wave is captured in the model (Fig. 3).

\section{2) SWEAD CONFIGURATION}

We configured a third-order nonlinear HOSM for a computational domain considering the predictable region (Wu 2004) or predictable zone (Qi et al. 2018), which is limited by observation period and wave dispersion. For observational data period $T$, each wave component has a predictable region roughly determined as $C_{g} T$, where $C_{g}$ is group velocity. We set the HOSM spatial domain to $32 \lambda_{p}$, covering the $25 \lambda_{p}$ predictable region corresponding to $50 T_{p}$ observational period.

For a given regularization parameter, the cost function decreases monotonically unless $\alpha$ is less than 0.005 when the optimization becomes unstable. In principle, $\alpha$ can be adaptively reduced in steps as the cost function reduces. In the unidirectional twin experiment, we kept $\alpha$ constant for each case to compare optimization efficiency. The values used were, $\alpha=1$, $0.5,0.1$, and 0.05 .
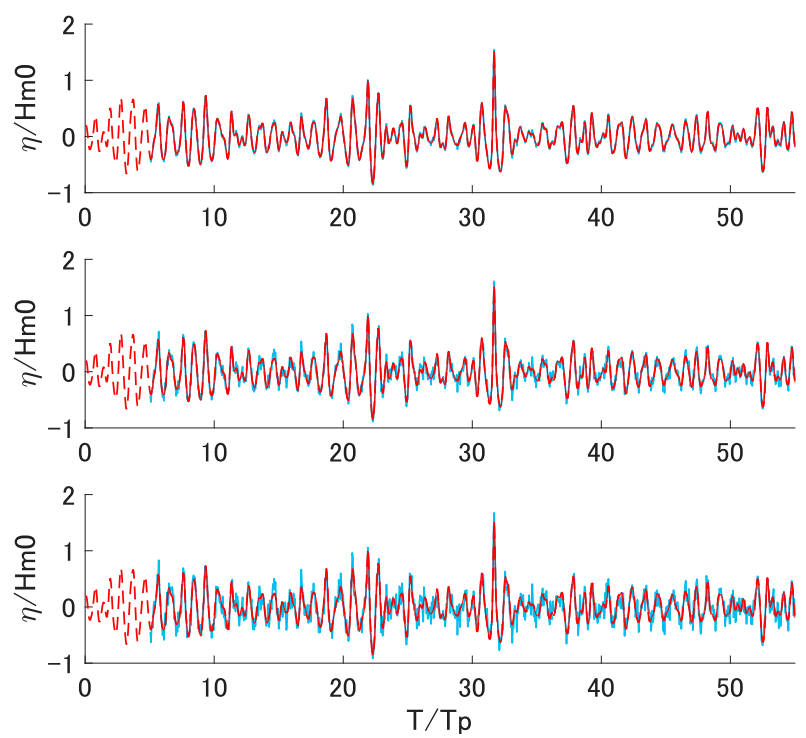

FIG. 4. Time series of virtual wave gauge data in the model. Red solid lines indicate the true time series extracted from true values. Light blue lines represent observational time series contaminated with random Gaussian noise: (top) 10\%, (middle) 30\%, and (bottom) $50 \%$. Data in the first $5 T_{p}$ nonlinear adjustment duration (red broken lines) were discarded in the twin experiment.

\section{3) RESUlts AND DISCUSSION}

The reconstructed wave field or the analysis $\left(\alpha_{\mathrm{opt}}=\right.$ $0.005, e_{\mathrm{TOL}}=0.2$ and $10 \%$ noise level in iteration 60 ) agreed very well with the truth or the virtual observations. Figure 5 shows a comparison of spatial waveform between virtual observations and analysis at a virtual wave gauge located in $x=0$. The freak wave group generating the freak wave at the wave gauge $\left(x / \lambda_{p}=0\right.$, $t / T_{p}=26$; Fig. 4) was located around $x / \lambda_{p}=16$ initially $\left(t / T_{p}=-5\right)$, see Fig. 3. Estimation around the freak wave group (around $x / \lambda_{p}=16$ of the initial wave; Fig. 5) was much improved (top panel) compared to the linear reconstruction (bottom panel). This comparison clearly demonstrates the usefulness of a nonlinear model to reconstruct the wave group containing a freak wave, allowing us to elucidate the underlying generation mechanism.

Figure 6 shows the spatiotemporal distribution of absolute error between the true and reconstructed values. Error is reduced in the predictable region bounded by the red broken lines; $x=0-25 \lambda_{p}$. Beyond the predictable region's edge, farther from observation point $x=0$, reconstructed values gradually reduce in magnitude. This is because Tikhonov regularization attenuates reconstructed waves outside of the predictable region where estimation is indeterminate. Waves are inevitably attenuated near model boundaries as well; therefore, regularization effectively mitigates artifacts of the HOSM's periodic boundary condition. 

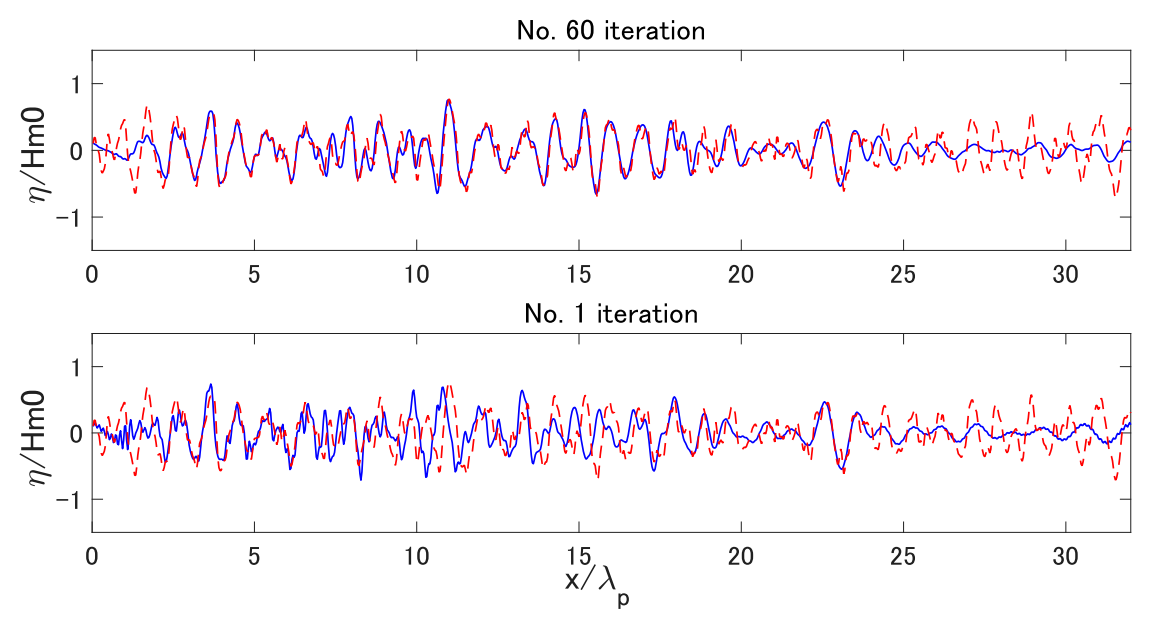

FIG. 5. Analysis wave profile (blue) of the unidirectional case's initial wave, with a $10 \%$ noise level. Red lines represent true values. (top) Analysis using the SWEAD method (nonlinear HOSM + a4DVar). (bottom) Linear reconstruction results.

Perturbation reuse (section 2c) accelerates the optimization process. Figure 7 shows cost function history for the $10 \%$ noise level case. Optimization with perturbation reuse (blue lines, $e_{\mathrm{TOL}}=0.2$ ) was faster than without perturbation reuse (red lines, $e_{\mathrm{TOL}}=0.0$ ). The total number of concatenated perturbations can increase and decrease to assure perturbation linearity, but there was an overall upward trend for the case of $e_{\mathrm{TOL}}=0.2$ (blue lines in Fig. 8). Larger numbers of concatenated perturbations were associated with faster optimization. However, if all perturbations were reused $\left(e_{\mathrm{TOL}}=\infty\right.$, black lines in Figs. 7 and 8), optimization diverged at an earlier stage because perturbations not conforming to the linear approximation were also used. The error tolerance $e_{\mathrm{TOL}}$ should be tuned by checking the balance between optimization speed and stability. The 50-ensemble optimization (solid lines) was faster than the 10-ensemble optimization (broken lines), which indicates that optimization speed can be further increased using massive parallel computation.

We examined wave reconstruction robustness by changing noise levels to $10 \%, 30 \%$, and $50 \%$. We selected $\alpha=0.005$ and $e_{\mathrm{TOL}}=0.2$ as tuning parameters. Figure 9 shows the histories of the cost function and Fig. 10 shows reconstructed initial waves. In all cases, wave fields were reproduced well in the predictable region, including the freak wave group around $x / \lambda_{p}=16$. Absolute error was sufficiently small even with noise levels of $30 \%$ and $50 \%$. Moreover, we evaluated the analysis' accuracy from a single realization in the predictable region (see Fig. 6 for the definition) using the root-mean-square error (RMSE) and correlation coefficient. In the $10 \%$, $30 \%$, and $50 \%$ noise cases, RMSEs were $14 \%, 16 \%$, and $15 \%$ of the significant wave height $H_{m 0}$, respectively, and correlation coefficients were $0.86,0.83$, and 0.84 , respectively. We conclude that the reconstruction has satisfactory accuracy and that the reconstruction is robust to noise.

\section{b. Multidirectional $3 D$ wave twin experiment}

\section{1) Problem Settings}

We extended the SWEAD method to analyze a directional wave field. Obviously, a single time series cannot be used to reconstruct a directional wave field because of directional ambiguity; therefore, a measurement with a directional spectrum is needed with a

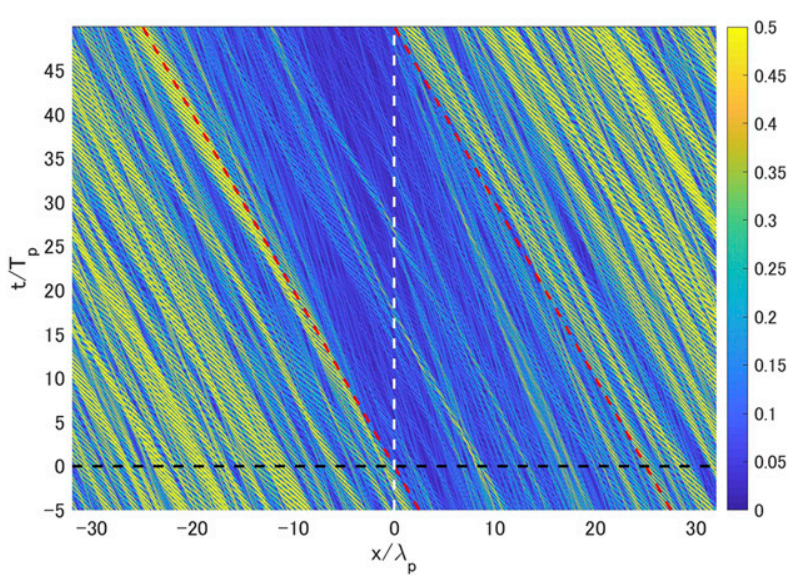

FIG. 6. Spatiotemporal absolute error between true value $\eta_{t}$ and reconstructed value $\eta_{r}$ at iteration 60 , with a $10 \%$ noise level. Wave gauge data correspond to the white broken line. The area beneath the horizontal black broken line is the nonlinear spinup period. The area enclosed by the red broken lines and black line is the predictable region. 


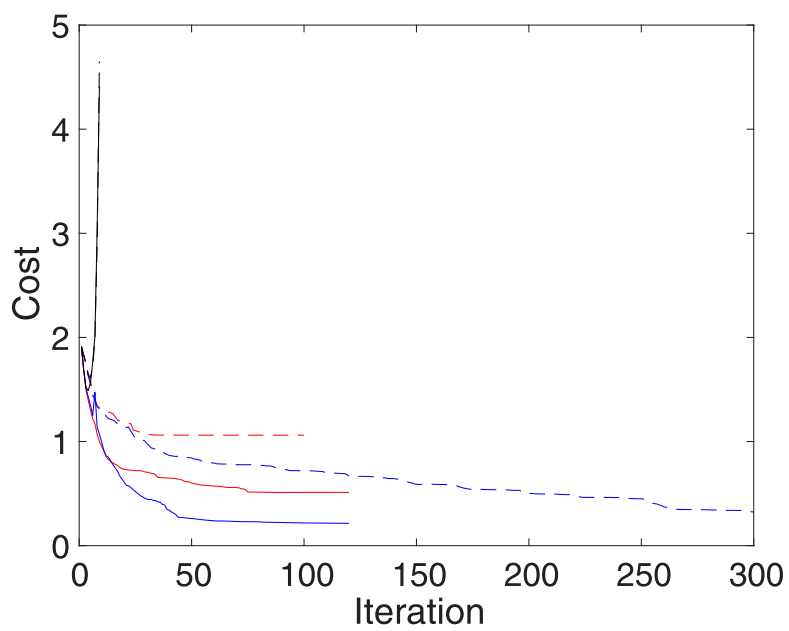

FIG. 7. History of the cost function in the unidirectional wave reconstruction, with a $10 \%$ noise level. Results of 50-ensemble (solid) and 10-ensemble (broken) assimilations are shown. Error tolerances were $e_{\mathrm{TOL}}=0.2$ (blue), $e_{\mathrm{TOL}}=0$ (without concatenation; red), and $e_{\mathrm{TOL}}=\infty$ (with full concatenation; black).

minimum of 3-degrees-of-freedom observation (LonguetHiggins et al. 1963). Here we consider a stereo camera imaging technique (Benetazzo 2006) for reconstructing a two-dimensional surface elevation wave field, which has already been demonstrated in various seas (Benetazzo et al. 2017) and is proven a viable and powerful alternative to conventional directional wave riders. This section assesses the performance of the SWEAD method on multidirectional sea conditions using virtual stereo reconstructed wave records.

We conducted twin experiments similar to a unidirectional case, and identified a freak wave in the true state using a Monte Carlo simulation. The third-order nonlinear HOSM generated a true state in a $64 \lambda_{p} \times 64 \lambda_{p}$ domain, which is large enough to mitigate periodic boundary condition effects (Fig. 11). The initial wave's

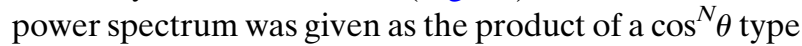
directional spreading function $(N=50)$, and we chose the frequency spectrum to be the same as that of the unidirectional case (JONSWAP spectrum, $\gamma=3.3$, and $\left.H_{m 0} k_{p} / 2=0.11\right)$. To test the performance of the SWEAD method when wave nonlinearity effects are strong, we selected a relatively severe sea condition with a narrower directionality than those in real sea conditions (e.g., Bitner-Gregersen and Toffoli 2012).

We extracted data imitating the stereo camera imaging from the true state from a $1 \lambda_{p} \times 1 \lambda_{p} \times 50 T_{p}$ domain containing a freak wave with a $1.53 H_{m 0}$ crest height (left column of Fig. 12). The selected freak waves' maximum crest was captured at the stereo camera observation's domain center. A $1 \lambda_{p} \times 1 \lambda_{p}$ domain size is typical for stereo camera imaging.

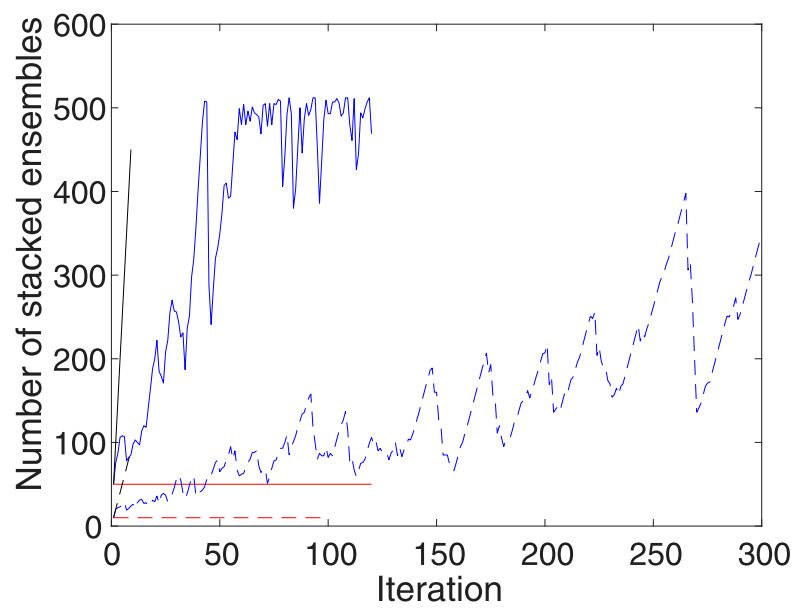

FIG. 8. Number of concatenated perturbations in the unidirectional wave reconstruction, with a $10 \%$ noise level. The results of 50-ensemble (solid) and 10-ensemble (broken) assimilations are shown. The error tolerances were $e_{\mathrm{TOL}}=0.2$ (blue), $e_{\mathrm{TOL}}=0$ (without concatenation; red), and $e_{\mathrm{TOL}}=\infty$ (with full concatenation; black).

\section{2) SWEAD CONFIGURATION}

We used a third-order HOSM with a $24 \lambda_{p} \times 24 \lambda_{p}$ domain size for wave reconstruction. We chose this domain size for the assimilation to cover the predictable region. In a strict sense, estimating the predictability region with directionality is rather complex, but in this test, we determined the region following the procedure outlined in the one-dimensional case. We set the concatenation error tolerance to $\mathrm{e}_{\mathrm{TOL}}=0.1$, and set the ensemble member number to 407 . Because the $2 \mathrm{D}$ problem had a large computational cost, we decreased the regularization parameter $\alpha$ in several steps during the optimization process

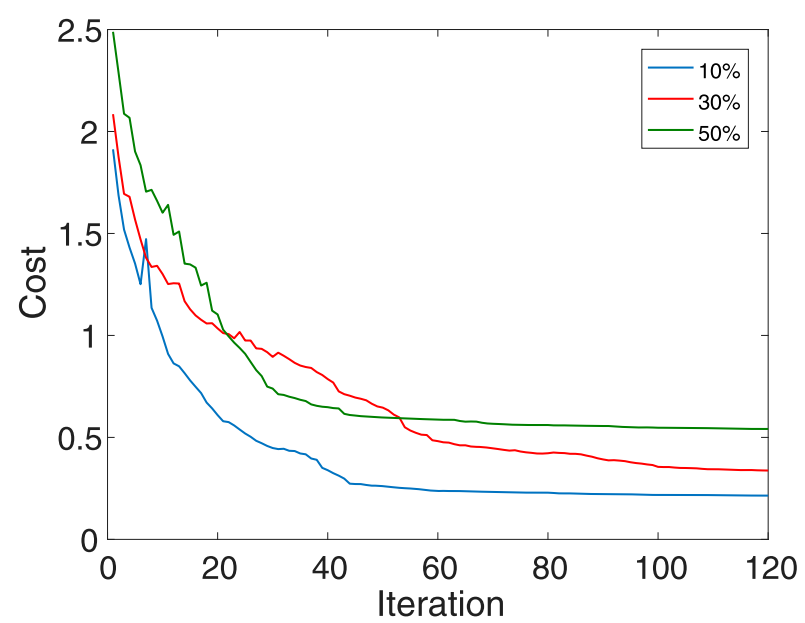

FIG. 9. History of cost functions with different noise levels. Tuning parameters were fixed at $\alpha=0.005$ and $e_{\mathrm{TOL}}=0.2$. 


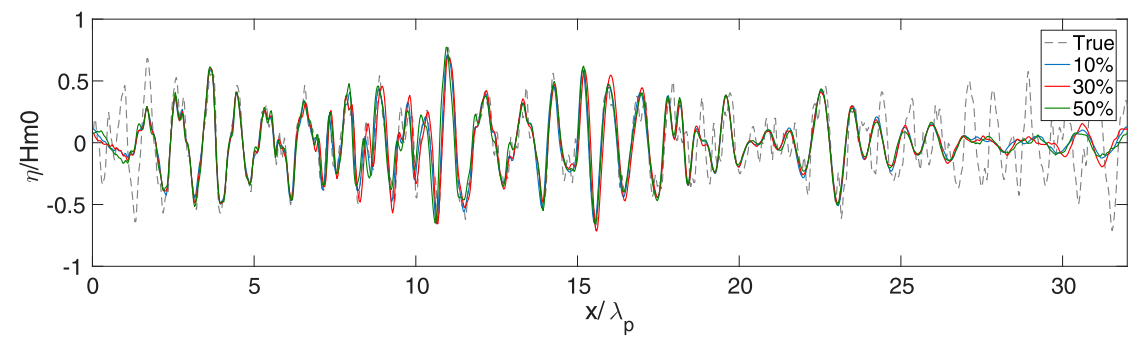

FIG. 10. Reconstructed initial waves at iteration 120. Legend shows noise percentage. The freak wave group was around $\mathbf{x} / \lambda_{p}=16$ at the initial wave $\left(t / T_{p}=-5\right)$ in Fig. 3.

for efficiency (Fig. 13). The a4DVar is a variant of the Gauss-Newton method (section 2b) known to be unstable immediately after starting the optimization process (Björck 1996). Therefore, we used relatively large values of regularization parameter $\alpha$ at the beginning of the optimization to stabilize optimization. As the cost function decreased, we decreased $\alpha$ in steps for a less-biased solution until the optimization became unstable [see section $4 \mathrm{a}(2)$ ]. The final stable regularization parameter values were $\alpha_{\text {opt }}=0.1$ for the $10 \%$ noise case, $\alpha_{\mathrm{opt}}=0.5$ for the $30 \%$ noise case, and $\alpha_{\text {opt }}=1$ for the $50 \%$ noise case. For a $10 \%$ noise level, the misfit $(1 / 2)\left[\mathbf{H}\left(\hat{\boldsymbol{\eta}}_{0}\right)-\mathbf{y}\right]^{\mathrm{T}} \mathbf{R}^{-1}\left[\mathbf{H}\left(\hat{\boldsymbol{\eta}}_{0}\right)-\mathbf{y}\right]$ squared error converged within 50 iterations (Fig. 13), and the optimization was terminated when the condition $J\left(\mathbf{x}_{0}^{n-1}\right)-J\left(\mathbf{x}_{0}^{n}\right)<0.003 \times J\left(\mathbf{x}_{0}^{0}\right)$ was satisfied. The estimation took $89 \mathrm{~h}$ using 12 Intel Xeon Phi 7250 processors of the University of Tokyo supercomputer Oakforest-PACS to conduct 407 ensemble simulations using HOSM M $=3$.

\section{3) RESUltS AND DISCUSSION}

Surface elevation truth and analyses (10\% noise level) are compared in Fig. 12. The freak wave group's propagation is captured in the red frames moving at a group velocity of the peak wavenumber component. To quantify the accuracy of the SWEAD method, we evaluated the correlation and RMSE of the analysis against true values within the frame following the wave group (Fig. 14). Maximum correlation was 0.90 and minimum RMSE was $15 \%$ of the significant wave height $H_{m 0}$. The freak wave group was reproduced well in directional seas. Regularization proved effective in directional wave fields, eliminating high wavenumber features and suppressing wave amplitudes outside the predictable region.

The SWEAD method assimilating virtual stereo camera imaging data well reconstructed the freak wave group. Figure 14 shows deviation of the analysis from observations and compares results with different noise levels. The $30 \%$ noise level case was as accurate as the $10 \%$ noise level case and was more accurate than the
$50 \%$ noise level case. In the $50 \%$ noise case, estimation degradation may have been affected by the choice of a larger regularization parameter. What is remarkable is that the high correlation values and low RMSE values were sustained for almost 10 wave periods in relatively low noise level cases $(10 \%$ and $30 \%)$. Although this comparison demonstrates that the SWEAD method's performance is within the predictability region, it also implies that the freak wave group maintained coherence for an extended time. Thus, including nonlinear dynamics is essential for reproducing such a wave group.

\section{Conclusions}

This study presented the SWEAD method to reproduce freak wave spatiotemporal profiles from observational data. The SWEAD method's novelties are 1) the application of a4DVAR to the HOSM and

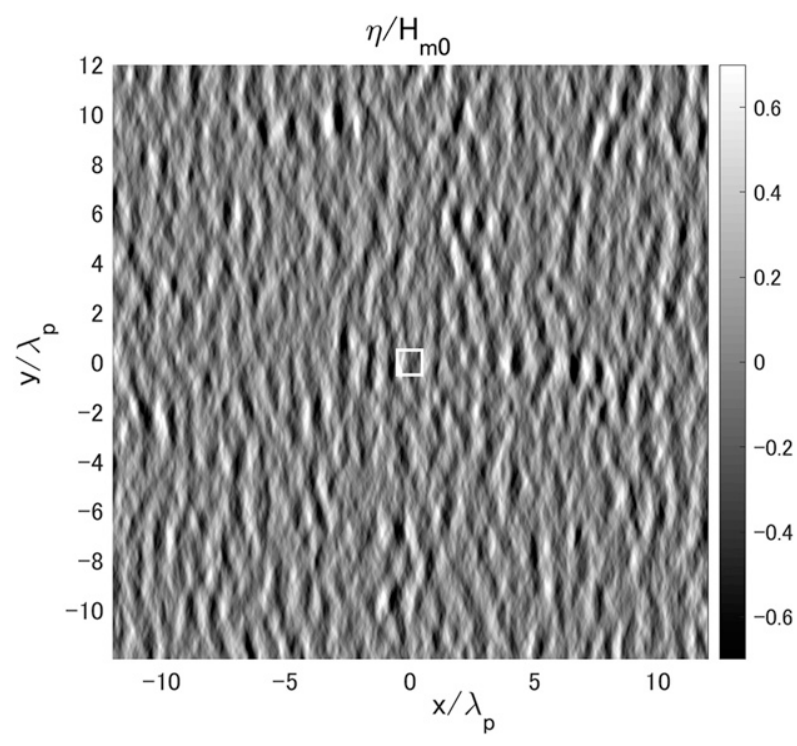

FIG. 11. Two-dimensional true initial wave field for the twin experiment shown in a $24 \lambda_{p}$ square area. Actual domain size was $64 \lambda_{p} \times 64 \lambda_{p}$. The observational area mimicking stereo imaging is shown in the center of the domain, $1 \lambda_{p} \times 1 \lambda_{p}$ (white square). 

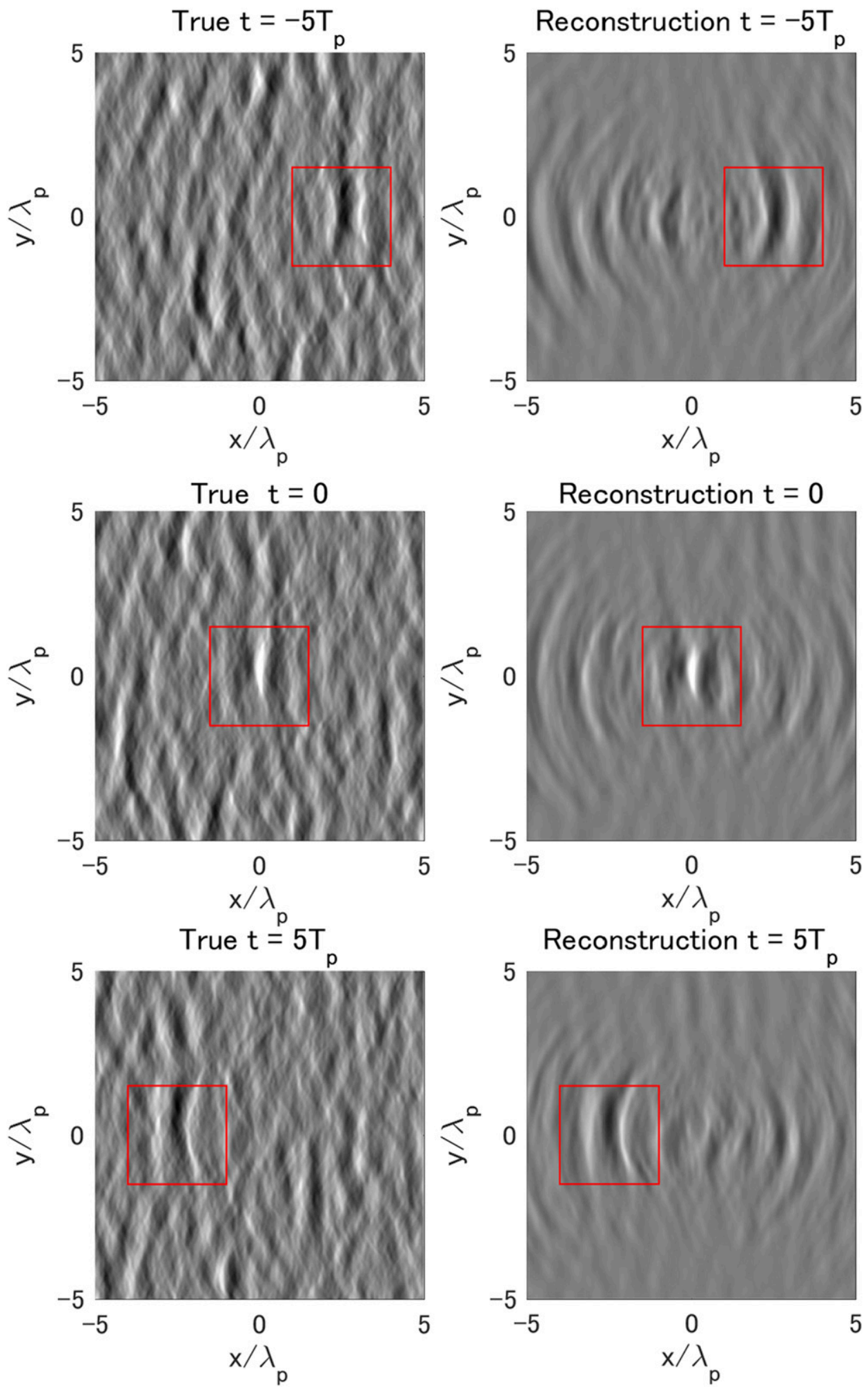

FIG. 12. Snapshots comparing (left) true and (right) reconstructed values from virtual stereo camera image data. The noise level was $10 \%$. Time $t=0$ corresponds to when the freak wave crest reached its maximum. The wave propagated from right to left. The red squares are $3 \lambda_{p} \times 3 \lambda_{p}$ frames moving at the spectral peak's group velocity. 


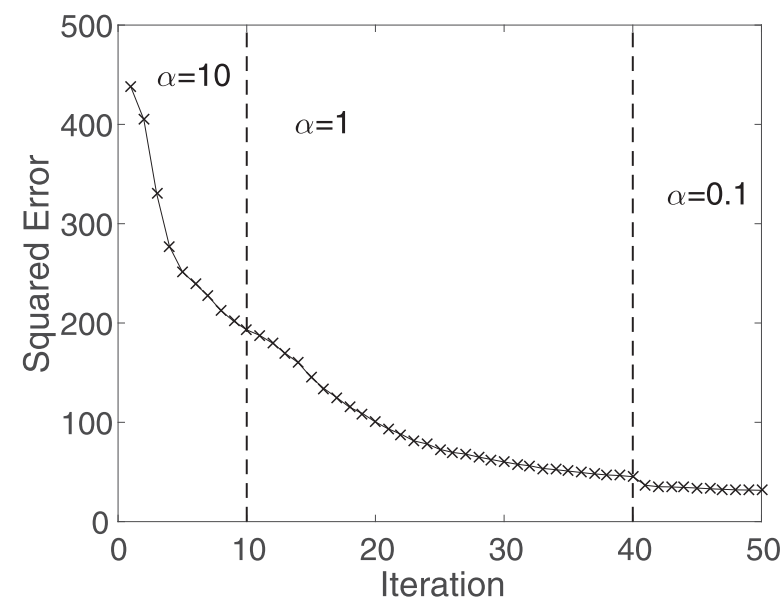

FIG. 13. Squared residual error between observational data and model prediction $(1 / 2)\left[\mathbf{H}\left(\mathbf{x}_{0}\right)-\mathbf{y}\right]^{\mathrm{T}} \mathbf{R}^{-1}\left[\mathbf{H}\left(\mathbf{x}_{0}\right)-\mathbf{y}\right]$ for nonlinear reconstruction at a $10 \%$ noise level. Regularization parameter $\alpha$ was reduced in steps: $\alpha=10$ for iterations $1-10, \alpha=1$ for iterations $11-40$, and $\alpha=0.1$ for iterations $41-50$.

2) the utilization of the background power spectrum for regularization.

\section{a. Application of a4DVar to the HOSM}

The SWEAD applies a4DVar to reconstruct waves using the HOSM. Although the HOSM is suitable for accurately solving nonlinear wave evolution with reasonable speed, implementing the adjoint code can be cumbersome when considering higher-order nonlinearity. a4DVar uses an ensemble simulation to compute the gradient; therefore, it requires far less coding effort than conventional adjoint methods. Parallelization to increase convergence speed is also much easier than with ordinary 4DVar.

We also proposed modifications to a4DVar. The SWEAD method chooses ensemble members from the misfit's power spectrum, whereas the original a4DVar chooses them from the misfit's EOF modes or from the model trajectory. The scheme to reuse initial condition perturbations was also modified by imposing a linear approximation constraint. As a result, unlimited reuse of perturbed data is possible, thereby boosting convergence speed. This approach is equivalent to imposing the secant condition on ensemble-based Newton optimization.

\section{b. Utilization of the background power spectrum for regularization}

The SWEAD method reflects prior power spectrum information on the regularization term in the cost function [Eq. (11)]. The background power spectrum obtained from observations or a wave model constrains
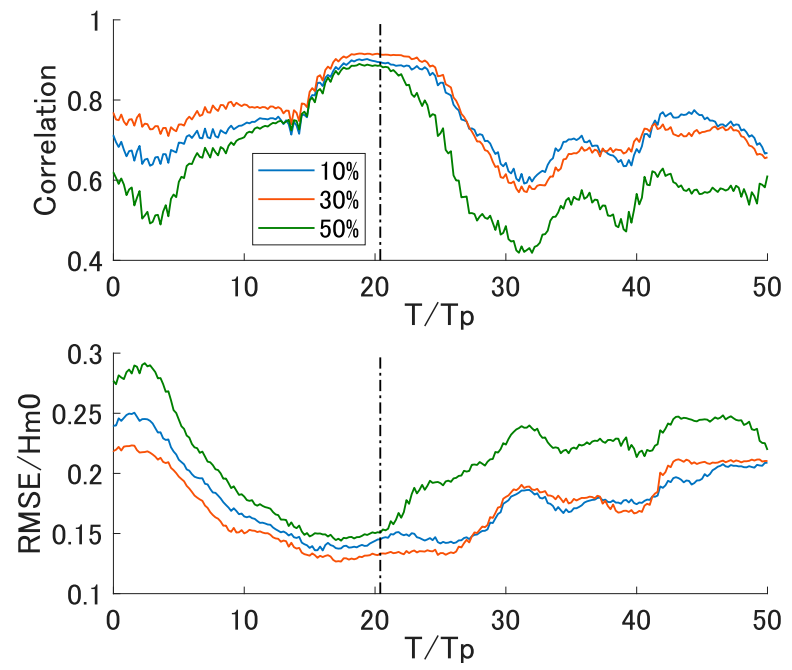

FIG. 14. Comparison of spatial snapshots of true and reconstructed values in a $3 \lambda_{p} \times 3 \lambda_{p}$ frame moving at group velocity. (top) Correlation coefficients and (bottom) root-mean-square error are compared. The vertical gray dash-dotted line indicates when the freak wave crest reached its maximum.

the reconstructed value's wave amplitude and stabilizes the optimization. Optimizing the cost function gives the initial wave's amplitude and phase from observational data $\mathbf{y}$ using the HOSM prediction $\mathbf{H}\left(\mathbf{x}_{0}\right)$ and the background power spectrum $S(\mathbf{k})$.

The SWEAD method analyzes observational data to estimate wave phases utilizing a third-generation wave model and the HOSM. Therefore, this method enhances previous methods combining third-generation wave models and the HOSM for estimating phase-averaged information, such as freak wave occurrence probability obtained by Monte Carlo simulations (Bitner-Gregersen et al. 2014; Trulsen et al. 2015; Fedele et al. 2016; Fujimoto et al. 2019). In this study, we tested the SWEAD method using twindata experiments with a unidirectional wave reconstructed from virtual wave gauge data and a multidirectional wave reconstructed from virtual stereo camera imaging data. In both cases, reconstructed values showed good agreement with true values. Thus, this study successfully demonstrated that nonlinear inversion of a freak wave is possible using the newly proposed SWEAD method.

Use of the SWEAD method is not limited to study of freak waves. The SWEAD method can also be useful for engineering purposes, such as assessing ship accident causes, assisting the design of offshore structures, and predicting waves for safe and efficient operations. For oceanographic purposes, the SWEAD method can be a useful extension to conventional phase-averaged spectral estimation, and can also be useful in experimental tanks, allowing reconstruction of an entire wave field from a limited amount of wave gauge data. 
The SWEAD method's advantage is that it does not require construction of adjoint code and thus does not depend on the selected numerical model. In this study, we used the HOSM without external forcings or dissipation. The HOSM has been extended to incorporate wind input and dissipation (Liu et al. 2010; Tian and Choi 2013), linear shear current (Guyenne 2017), and bathymetry effects (Gouin et al. 2016). Freak wave generation is known to be affected by current (e.g., Onorato et al. 2011; Toffoli et al. 2013, 2015) and wind (e.g., Toffoli et al. 2017), thus these physical processes should be incorporated, which can be easily done with the SWEAD method.

Acknowledgments. The authors acknowledge M. Yaremchuk, who had given us valuable advice upon completing the final version of the manuscript. W.F. acknowledges support from the Fundamental Research Development Association for Shipbuilding and Offshore (REDAS), Japan. This research was funded by a Japan Society for the Promotion of Science (JSPS) Grant-in-Aid for Scientific Research (KAKENHI), Grant 16H02429.

\section{APPENDIX}

\section{Evaluating Deviation from the Linear Approximation in Perturbed Simulations}

The secant condition is a key concept in continuous optimization and is used to derive an approximation of the Hessian matrix in quasi-Newton methods (e.g., Liu and Nocedal 1989). Similarly, in this study we use the secant condition to evaluate the accuracy of perturbations in terms of the linear approximation.

The secant condition states that the product of the Hessian matrix of the cost function $\tilde{\mathbf{H}}=\mathbf{H}^{\mathrm{T}} \mathbf{R}^{-1} \mathbf{H}+\mathbf{B}^{-1}$ and iterative update of the initial condition $\mathbf{x}_{0}^{n+1}-\mathbf{x}_{0}^{n}$ should equal the increment of the cost function's gradient. The secant condition of the cost function [Eq. (1)] reads

$$
\left(\mathbf{H}^{\mathrm{T}} \mathbf{R}^{-1} \mathbf{H}+\mathbf{B}^{-1}\right)\left(\mathbf{x}_{0}^{n+1}-\mathbf{x}_{0}^{n}\right)=\nabla J\left(\mathbf{x}_{0}^{n+1}\right)-\nabla J\left(\mathbf{x}_{0}^{n}\right) .
$$

By multiplying $\mathbf{P}^{\mathrm{T}}$ into Eq. (A1) from the left and then substituting Eq. (7), the above equation can be rewritten as

$$
\begin{aligned}
\mathbf{P}^{\mathrm{T}}\left(\mathbf{H}^{\mathrm{T}} \mathbf{R}^{-1} \mathbf{H}+\mathbf{B}^{-1}\right)\left(\mathbf{x}_{0}^{n+1}-\mathbf{x}_{0}^{n}\right)= & \boldsymbol{\delta} \mathbf{Y}^{\mathrm{T}} \mathbf{R}^{-1}\left[\mathbf{H}\left(\mathbf{x}_{0}^{n+1}\right)-\mathbf{y}\right]+\mathbf{P}^{\mathrm{T}} \mathbf{B}^{-1} \mathbf{x}_{0}^{n+1} \\
& -\boldsymbol{\delta} \mathbf{Y}^{\mathrm{T}} \mathbf{R}^{-1}\left[\mathbf{H}\left(\mathbf{x}_{0}^{n}\right)-\mathbf{y}\right]+\mathbf{P}^{\mathrm{T}} \mathbf{B}^{-1} \mathbf{x}_{0}^{n}+O\left(\left\|\mathbf{s}^{n}\right\|^{2}\right) .
\end{aligned}
$$

An iterative update of the initial condition is given as a linear superposition of perturbation vectors, $\mathbf{x}_{0}^{n+1}=$ $\mathbf{x}_{0}^{n}+\mathbf{P s}^{n}$ :

$$
\begin{aligned}
\mathbf{P}^{\mathrm{T}}\left(\mathbf{H}^{\mathrm{T}} \mathbf{R}^{-1} \mathbf{H}+\mathbf{B}^{-1}\right) \mathbf{P} \mathbf{s}^{n} & =\left(\boldsymbol{\delta} \mathbf{Y}^{\mathrm{T}} \mathbf{R}^{-1} \boldsymbol{\delta} \mathbf{Y}+\mathbf{P}^{\mathrm{T}} \mathbf{B}^{-1} \mathbf{P}\right) \mathbf{s}^{n} \\
& =\boldsymbol{\delta} \mathbf{Y}^{\mathrm{T}} \mathbf{R}^{-1}\left[\mathbf{H}\left(\mathbf{x}_{0}^{n+1}\right)-\mathbf{H}\left(\mathbf{x}_{0}^{n}\right)\right]+\mathbf{P}^{\mathrm{T}} \mathbf{B}^{-1} \mathbf{P s}^{n}+O\left(\left\|\mathbf{s}^{n}\right\|^{\mathbf{2}}\right)
\end{aligned}
$$

We used Eq. (4) in the last transformation. Now, the residual $O\left(\left\|\mathbf{s}^{n}\right\|^{2}\right)$ is incorporated into $\mathbf{s}^{n^{\prime}}$ :

$$
\begin{aligned}
\left(\boldsymbol{\delta} \mathbf{Y}^{\mathrm{T}} \mathbf{R}^{-1} \boldsymbol{\delta} \mathbf{Y}+\mathbf{P}^{\mathrm{T}} \mathbf{B}^{-1} \mathbf{P}\right) \mathbf{s}^{n^{\prime}}= & \boldsymbol{\delta} \mathbf{Y}^{\mathrm{T}} \mathbf{R}^{-1}\left[\mathbf{H}\left(\mathbf{x}_{0}^{n+1}\right)-\mathbf{H}\left(\mathbf{x}_{0}^{n}\right)\right] \\
& +\mathbf{P}^{\mathrm{T}} \mathbf{B}^{-1} \mathbf{P} \mathbf{s}^{n} .
\end{aligned}
$$

Equation (8) can be derived by inverting the above equation. If the system is linear, then $\mathbf{H}\left(\mathbf{x}_{0}^{n}\right)=\mathbf{H} \mathbf{x}_{0}^{n}$ and $\mathbf{s}^{n^{\prime}}=\mathbf{s}^{n}$ because the residual $O\left(\left\|\mathbf{p}_{m}\right\|^{2}\right)$ is zero in Eq. (3). Therefore, the residual $O\left(\left\|\mathbf{s}^{n}\right\|^{2}\right)$ in Eq. (A3) is also zero. However, when the system is nonlinear, the residual $O\left(\left\|\mathbf{s}^{n}\right\|^{2}\right)$ is not negligible and $\mathbf{s}^{n^{\prime}}$ thus deviates from $\mathbf{s}^{n}$.

\section{REFERENCES}

Annenkov, S. Y., and V. I. Shrira, 2009: Evolution of kurtosis for wind waves. Geophys. Res. Lett., 36, L13603, https://doi.org/ 10.1029/2009GL038613.

Aragh, S., and O. Nwogu, 2008: Variation assimilating of synthetic radar data into a pseudo-spectral wave model. J. Coastal Res., 2008, 235-244, https://doi.org/10.2112/1551-5036-52.sp1.235.

,$- \ldots$, and D. Lyzenga, 2008: Improved estimation of ocean wave fields from marine radars using data assimilation techniques. 18th Int. Offshore Polar Engineering Conf., Vancouver, BC, Canada, International Society of Offshore and Polar Engineers, ISOPE-I-08-300.

Benetazzo, A., 2006: Measurements of short water waves using stereo matched image sequences. Coastal Eng., 53, 1013-1032, https://doi.org/10.1016/j.coastaleng.2006.06.012. 
- and Coauthors, 2017: On the shape and likelihood of oceanic rogue waves. Sci. Rep., 7, 8276, https://doi.org/10.1038/s41598017-07704-9.

Bitner-Gregersen, E. M., and A. Toffoli, 2012: On the probability of occurrence of rogue waves. Nat Hazards Earth Syst. Sci., 12, 751-762, https://doi.org/10.5194/nhess-12-751-2012.

— , and Coauthors, 2014: The North Sea Andrea storm and numerical simulations. Nat. Hazards Earth Syst. Sci., 14, 14071415, https://doi.org/10.5194/nhess-14-1407-2014.

Björck, A., 1996: Nonlinear least squares problems. Numerical Methods for Least Squares Problems, Society for Industrial and Applied Mathematics, 339-358.

Blondel-Couprie, E., F. Bonnefoy, and P. Ferrant, 2010: Deterministic non-linear wave prediction using probe data. Ocean Eng., 37, 913-926, https://doi.org/10.1016/j.oceaneng.2010.03.002.

,-- , and -2013 : Experimental validation of non-linear deterministic prediction schemes for long-crested waves. Ocean Eng., 58, 284-292, https://doi.org/10.1016/j.oceaneng.2012.10.014.

Dommermuth, D. G., 2000: The initialization of nonlinear waves using an adjustment scheme. Wave Motion, 32, 307-317, https://doi.org/10.1016/S0165-2125(00)00047-0.

— study of nonlinear gravity waves. J. Fluid Mech., 184, 267-288, https://doi.org/10.1017/S002211208700288X.

Fedele, F., and Coauthors, 2016: Real world ocean rogue waves explained without the modulational instability. Sci. Rep., 6, 27715, https://doi.org/10.1038/srep27715.

Flanagan, J. D., and Coauthors, 2016: Extreme water waves off the west coast of Ireland: Analysis of ADCP measurements. 26th Int. Ocean and Polar Engineering Conf., Rhodes, Greece, International Society of Offshore and Polar Engineers, 589.

Fonseca, N., C. Guedes Soares, and R. Pascoal, 2006: Structural loads induced in a containership by abnormal wave conditions. J. Mar. Sci. Technol., 11, 245-259, https://doi.org/10.1007/ s00773-006-0222-9.

Fujimoto, W., T. Waseda, and A. Webb, 2019: Impact of the fourwave quasi-resonance on freak wave shapes in the ocean. Ocean Dyn., 69, 101-121, https://doi.org/10.1007/s10236-0181234-9.

Gouin, M., G. Ducrozet, and P. Ferrant, 2016: Development and validation of a non-linear spectral model for water waves over variable depth. Eur. J. Mech., 57B, 115-128, https://doi.org/ 10.1016/j.euromechflu.2015.12.004.

Gramstad, O., and K. Trulsen, 2007: Influence of crest and group length on the occurrence of freak waves. J. Fluid Mech., 582, 463-472, https://doi.org/10.1017/S0022112007006507.

Guedes Soares, C., N. Fonseca, and R. Pascoal, 2008: Abnormal wave-induced load effects in ship structures. J. Ship Res., 52, $30-44$.

Guyenne, P., 2017: A high-order spectral method for nonlinear water waves in the presence of a linear shear current. Comput. Fluids, 154, 224-235, https://doi.org/10.1016/j.compfluid.2017.06.004.

Haver, S., 2004: A possible freak wave event measured at the Draupner jacket January 1 1995. Rogue Waves 2004, Brest, France, Ifremer.

Houtani, H., K. Tanizawa, T. Waseda, and H. Sawada, 2016: An experimental investigation on the influence of the temporal variation of freak wave geometry on the elastic response of a container ship. Third Int. Conf. on Violent Flows, Osaka, Japan, Japan Society of Naval Architects and Ocean Engineers, 9-11.

Janssen, P. A. E. M., 2003: Nonlinear four-wave interactions and freak waves. J. Phys. Oceanogr., 33, 863-884, https://doi.org/ 10.1175/1520-0485(2003)33<863:NFIAFW > 2.0.CO;2
Kharif, C., and E. Pelinovsky, 2003: Physical mechanisms of the rogue wave phenomenon. Eur. J. Mech., 22B, 603-634, https:// doi.org/10.1016/j.euromechflu.2003.09.002.

Liu, C., Q. Xiao, and B. Wang, 2008: An ensemble-based fourdimensional variational data assimilation scheme. Part I: Technical formulation and preliminary test. Mon. Wea. Rev., 136, 3363-3373, https://doi.org/10.1175/2008MWR2312.1.

,-- , and -2009 : An ensemble-based four-dimensional variational data assimilation scheme. Part II: Observing system simulation experiments with Advanced Research WRF (ARW). Mon. Wea. Rev., 137, 1687-1704, https://doi.org/ 10.1175/2008MWR2699.1.

Liu, D. C., and J. Nocedal, 1989: On the limited memory BFGS method for large scale optimization. Math. Program., 45, 503528, https://doi.org/10.1007/BF01589116.

Liu, Y., D. Yang, X. Guo, and L. Shen, 2010: Numerical study of pressure forcing of wind on dynamically evolving water waves. Phys. Fluids, 22, 041704, https://doi.org/10.1063/1.3414832.

Longuet-Higgins, M. S., D. E. Cartwright, and N. D. Smith, 1963: Observations of the directional spectrum of sea waves using the motions of a floating buoy. Ocean Wave Spectra, PrenticeHall, 111-136.

Magnusson, A. K., and M. A. Donelan, 2013: The Andrea wave characteristics of a measured North Sea rogue wave. J. Offshore Mech. Arct. Eng., 135, 031108, https://doi.org/ 10.1115/1.4023800.

Onorato, M., A. R. Osborne, and M. Serio, 2002: Extreme wave events in directional, random oceanic sea states. Phys. Fluids, 14, L25-L28, https://doi.org/10.1063/1.1453466.

- D. Proment, and A. Toffoli, 2011: Triggering rogue waves in opposing currents. Phys. Rev. Lett., 107, 184502, https:// doi.org/10.1103/PhysRevLett.107.184502.

Panteleev, G., M. Yaremchuk, and W. E. Rogers, 2015: Adjointfree variational data assimilation into a regional wave model. J. Atmos. Oceanic Technol., 32, 1386-1399, https://doi.org/ 10.1175/JTECH-D-14-00174.1.

Qi, Y., G. Wu, Y. Liu, and D. K. P. Yue, 2018: Predictable zone for phase-resolved reconstruction and forecast of irregular waves. Wave Motion, 77, 195-213, https://doi.org/10.1016/ j.wavemoti.2017.12.001.

Slunyaev, A., E. Pelinovsky, and C. Guedes Soares, 2013: Reconstruction of extreme events through numerical simulations. J. Offshore Mech. Arct. Eng., 136, 011302, https://doi.org/10.1115/ 1.4025545 .

Socquet-Juglard, H., and Coauthors, 2005: Probability distributions of surface gravity waves during spectral changes. J. Fluid Mech., 542, 195-216, https://doi.org/10.1017/S0022112005006312.

Tanaka, M., and N. Yokoyama, 2004: Effects of discretization of the spectrum in water-wave turbulence. Fluid Dyn. Res., 34, 199-216, https://doi.org/10.1016/j.fluiddyn.2003.12.001.

Tian, Z., and W. Choi, 2013: Evolution of deep-water waves under wind forcing and wave breaking effects: Numerical simulations and experimental assessment. Eur. J. Mech., 41B, 11-22, https://doi.org/10.1016/j.euromechflu.2013.04.001.

— M. Perlin, and W. Choi, 2010: Energy dissipation in twodimensional unsteady plunging breakers and an eddy viscosity model. J. Fluid Mech., 655, 217-257, https://doi.org/10.1017/ S0022112010000832.

Toffoli, A., and E. M. Bitner-Gregersen, 2011: Extreme and rogue waves in directional wave fields. Open Ocean Eng. J., 6, 24-33, https://doi.org/10.2174/1874835x01104010024.

- and Coauthors, 2013: Excitation of rogue waves in a variable medium: An experimental study on the interaction of water 
waves and currents. Phys. Rev., 87E, 051201, https://doi.org/ 10.1103/Physreve.87.051201.

—_, and Coauthors, 2015: Rogue waves in opposing currents: An experimental study on deterministic and stochastic wave trains. J. Fluid Mech., 769, 277-297, https://doi.org/10.1017/ jfm.2015.132.

— annular wave flume. Phys. Rev. Lett., 118, 144503, https:// doi.org/10.1103/PhysRevLett.118.144503.

Tolman, H. L., and Coauthors 2016: User manual and system documentation of WAVEWATCH III. NCEP Tech. Note, $361 \mathrm{pp}$.

Trulsen, K., and Coauthors, 2015: Crossing sea state and rogue wave probability during the Prestige accident. J. Geophys. Res. Oceans, 120, 7113-7136, https://doi.org/10.1002/2015JC011161.

van Groesen, E., and A. P. Wijaya, 2017: High waves in Draupner seas-Part 2: Observation and prediction from synthetic radar images. J. Ocean Eng. Mar. Energy, 3, 325-332, https://doi.org/ 10.1007/s40722-017-0090-x.

Waseda, T., and Coauthors, 2011: GPS-based wave observation using a moored oceanographic buoy in the deep ocean. 21st Int. Offshore Polar Eng. Conf., Maui, HI, International Society of Offshore and Polar Engineers, ISOPE-I11-393.

West, B. J., and Coauthors, 1987: A new numerical method for surface hydrodynamics. J. Geophys. Res., 92, $11803-11824$, https://doi.org/10.1029/JC092iC11p11803.

White, B. S., and B. Fornberg, 1998: On the chance of freak waves at sea. J. Fluid Mech., 355, 113-138, https://doi.org/10.1017/ S0022112097007751.

Wijaya, A. P., P. Naaijen, Andonowati, and E. van Groesen, 2015: Reconstruction and future prediction of the sea surface from radar observations. Ocean Eng., 106, 261-270, https://doi.org/ 10.1016/j.oceaneng.2015.07.009.

Willoughby, R. A., 1979: Solutions of ill-posed problems (A. N. Tikhonov and V. Y. Arsenin). SIAM Rev., 21, 266-267, https:// doi.org/10.1137/1021044.
Wu, G., 2004: Direct simulation and deterministic prediction of large-scale nonlinear ocean wave-field. Ph.D. thesis, Massachusetts Institute of Technology, $258 \mathrm{pp}$.

Xiao, W., Y. Liu, G. Wu, and D. K. P. Yue, 2013: Rogue wave occurrence and dynamics by direct simulations of nonlinear wave-field evolution. J. Fluid Mech., 720, 357-392, https:// doi.org/10.1017/jfm.2013.37.

Yaremchuk, M., D. Nechaev, and G. Panteleev, 2009: A method of successive corrections of the control subspace in the reducedorder variational data assimilation. Mon. Wea. Rev., 137, 2966-2978, https://doi.org/10.1175/2009MWR2592.1.

— , P. Martin, A. Koch, and C. Beattie, 2016: Comparison of the adjoint and adjoint-free 4DVar assimilation of the hydrographic and velocity observations in the Adriatic Sea. Ocean Modell., 97, 129-140, https://doi.org/10.1016/j.ocemod.2015.10.010.

, G. Panteleev, C. Beattie, and A. Koch, 2017a: Adjointfree 4D variational data assimilation into regional models. Data Assimilation for Atmospheric, Oceanic and Hydrologic Applications, Vol. III, Springer, 83-114.

,$- \ldots$, and C. Beattie, 2017b: A hybrid approach to generating search subspaces in dynamically constrained 4-dimensional data assimilation. Ocean Modell., 117, 41-51, https://doi.org/ 10.1016/j.ocemod.2017.08.003.

Yoon, S., J. Kim, and W. Choi, 2015: An explicit data assimilation scheme for a nonlinear wave prediction model based on a pseudo-spectral method. IEEE J. Ocean Eng., 41, 112-122, https://doi.org/10.1109/JOE.2015.2406471.

Zakharov, V. E., 1968: Stability of periodic waves of finite amplitude on the surface of a deep fluid. J. Appl. Mech. Tech. Phys., 9, 190-194, https://doi.org/10.1007/BF00913182.

Zhu, J., H. Wang, and M. Kamachi, 2002: The improvement made by a modified TLM in 4DVar with a geophysical boundary layer model. Adv. Atmos. Sci., 19, 563-582, https://doi.org/ 10.1007/s00376-002-0001-4.

Zupanski, M., 2005: Maximum likelihood ensemble filter: Theoretical aspects. Mon. Wea. Rev., 133, 1710-1726, https:// doi.org/10.1175/MWR2946.1. 